\title{
Performance of the Locally Optimum Threshold Receiver and Several Suboptimum Nonlinear Receivers for ELF Noise
}

\author{
RAYMOND F. INGRAM, MEMBER, IEEE \\ (Invited Paper)
}

\begin{abstract}
Naturally occurring atmospheric noise encountered at the input to an extremely low frequency (ELF) receiver is non-Gaussian, consisting of the sum of a low-level Gaussian background and strong narrow pulses caused by single lightning strokes from nearby thunderstorms. It has been khown for a number of years that significant improvements in performance can be achieved if the non-Gaussian nature of the noise is considered when the ELF receiver is designed. The optimum receiver structure for detecting known threshold signals in additive white non-Gaussian noise is known to be the same as that which should be used if the noise were Gaussian, except that a zero-memory nonlinearity is placed between the receiver input and the Gaussian detector. The input-output characteristic of the nonlinearity is given by $-d / d x$ [In $\left.p_{n}(x)\right]$, where $p_{n}(x)$ is the first-order amplitude probability density function of the additive noise.

In this paper, the performance of this receiver, as well as of those where the nonlinearity is replaced by either a clipper, hole puncher, or hard limiter, is analytically evaluated for Middleton's Class B Noise Model. Although this analytical approach is different than the earlier empirical approach taken by Evans and Griffiths, it reaches identical conclusions concerning the design choice of the nonlinear noise processor for use at ELF. Briefly, it confirms that the optimum nonlinearity can achieve an improvement of as much as $\mathbf{2 0} \mathrm{dB}$ in effective signal-to-noise ratio. Moreover, it shows that the more practical clipper achieves an improvement typically within $0.5 \mathrm{~dB}$ of the optimum nonlinearity.
\end{abstract}

\section{INTRODUCTION}

A TMOSPHERIC noise in the extremely low-frequency (ELF) band of $20-500 \mathrm{~Hz}$ is caused by lightning strokes occurring worldwide [1]-[3]. A time history of this noise includes 1) a Gaussian background (which is the summation of distant lightning strokes attenuated and stretched in time by propagation through the earth-ionosphere waveguide) and 2) strong narrow pulses caused by single lightning strokes from nearby thunderstorms. The resulting noise at the input of an ELF receiver is distinctly non-Gaussian. Significant improvements in performance can be achieved if the non-Gaussian nature of this noise is considered when the receiver is designed.

The optimum receiver structure for detecting known threshold signals (i.e., low signal-to-noise ratio (SNR)) in additive white non-Gaussian noise is the same as that which should be used if the noise were Gaussian, except that a zero-memory nonlinearity should precede it. The input-output characteristic of the nonlinearity is given by $-d / d x\left[\ln p_{n}(x)\right]$, where $p_{n}(x)$ is the amplitude probability density function of the noise. In practice, the locally optimum nonlinearity is often replaced by a more easily implemented suboptimum nonlinearity, such as a

Manuscript received October 17, 1983; revised April 20, 1984.

The author is with the Naval Underwater Systems Center, New London Laboratory, New London, CT 06320. clipper, hole puncher, or hard limiter. The input-output characteristics of these suboptimum nonlinearities are shown in Fig. 1.

The receivers presently in service for the U.S. Navy experimental ELF program, which is known as the System Evaluation Operations (SVO), and the receiver currently under development for the operational ELF system both use the adaptive nonlinear noise processor and associated filtering that was first developed by M.I.T./Lincoln Laboratory in the early 1970's [4]-[6]. This design was based on the results of an empirical study that used digital tapes of time histories of high-level ELF atmospheric noise from several submarine operating areas.

In this paper, the improvements gained in the detection of known signals in additive white non-Gaussian noise by the use of a locally optimum threshold receiver (LOTR) and several suboptimum receivers are examined. The improvements are evaluated by numerically integrating the mathematical expressions of the receiver performance for a noise model that appears to adequately describe ELF noise. This analytical approach is entirely different than the earlier empirical approach taken by Evans and Griffiths [4]-[6]. The most significant contribution of this study is the comparison of the performance of receivers with the simple suboptimum nonlinearities to the performance of the LOTR.

\section{OPTIMUM RECEIVER STRUCTURES}

The locally optimum receiver structure for the threshold detection of binary coherent signals in an additive white noise environment is well known [7]-[9]. The derivation of this structure is summarized here to emphasize its primary assumptions.

The receiver structure is derived for a memoryless channel, in which the source output under hypothesis $H_{1}$ is a known signal $s(t)$, and under hypothesis $H_{0}$ is zero. Before observation, these source outputs are corrupted by an additive noise $n(t)$. The corrupted waveforms are then sampled uniformly to obtain $N$ samples. Each noise sample is a zero mean random variable $n_{i}$ with a variance $\sigma_{n}{ }^{2}$ and a first-order amplitude probability density function $p_{n}\left(x_{i}\right)$. The noise samples are assumed to be independent identically distributed random variables and are independent of the source output. The observations under the two hypotheses are

$$
\begin{array}{ll}
H_{1}: z_{i}=s_{i}+n_{i}, & i=1,2, \cdots, N \\
H_{0}: z_{i}=n_{i}, & i=1,2, \cdots, N .
\end{array}
$$




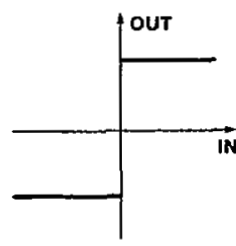

(a)

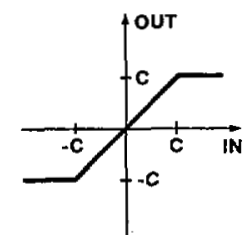

(b)

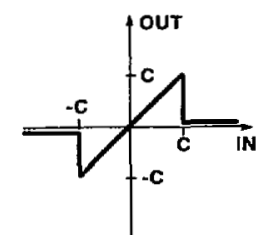

(c)
Fig. 1. Input-output characteristics of simple suboptimum nonlinearities. (a) Hard limiter. (b) Clipper. (c) Hole puncher.

The receiver that optimizes performance chooses between $H_{1}$ and $\dot{H}_{0}$, by comparing $p_{z / H_{1}}\left(z / H_{1}\right)$ with $p_{z / H_{0}}\left(z / H_{0}\right)$ relative to some constant threshold $\lambda$ which is determined by the $a$ priori probabilities of $H_{1}$ and $H_{0}$ and an "optimality criterion."

Because the additive noise samples are independent identically distributed random variables and the signal components are completely known, the conditional probability density function of the received vectors $z$ can be expressed in terms of the density functions of the individual noise components $p_{n}\left(n_{i}\right)$. Hence, the receiver uses the following likelihood ratio as its test statistic:

$$
\ln \Lambda(z)=\sum_{i=1}^{N}\left[\ln p_{n}\left(z_{i}-s_{i}\right)\right]-\sum_{i=1}^{N}\left[\ln p_{n}\left(z_{i}\right)\right] \underset{H_{0}}{\stackrel{H_{1}}{\gtrless}} \ln \lambda .
$$

When the operations of (1) are performed, one form of the optimum receiver has been implemented; there are, however, other possible optimum structures that, by definition, would yield equal performance. If, for example, each of the terms within the sums of (1) is expanded into an infinite Taylor series about $z_{i}$, the likelihood ratio test becomes

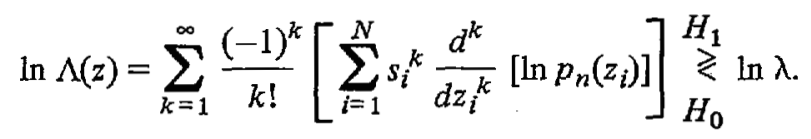

The optimum receiver, then, consists of a weighted sum of an infinite number of paths. Each path is made up of a matched filter preceded by a zero-memory nonlinear device. The $j$ th path has the form of a filter matched to the $j$ th power of the signal; this filter is preceded by a nonlinearity with an inputoutput transfer function given by the $j$ th derivative of the log. arithm of the first order amplitude probability density function of the noise. This receiver structure is interesting, because it clearly reduces to the expected structure for Gaussian noise and to a simple structure for threshold detection.

For the case of threshold detection, the input signal is sufficiently small to ensure that the higher order terms of $s_{i}$ in (2) are negligible relative to the first two terms. The second term has been shown to include a bias term that does not become small for large values of $N[10]$. This bias term does not change the spacing or the probability density functions of the test statistic under the two hypotheses but equivalently results in a new threshold, which will be denoted as $\lambda_{1}$. If a Neyman-Pearson test is used, as in the U.S. Navy ELF receiver, it is not necessary to calculate this second term. The likelihood ratio test then reduces to

$$
\ln \Lambda(z)=\sum_{i=1}^{N} s_{i}\left[-\frac{d}{d z_{i}}\left[\ln p_{n}\left(z_{i}\right)\right]\right] \underset{H_{0}}{\stackrel{H_{1}}{\gtrless}} \ln \lambda_{1}
$$

resulting in the receiver block diagram shown in Fig. 2. Hence, for the case of threshold detection of known signals in independent identically distributed noise samples, the locally optimum receiver structure is a correlation receiver preceded by a zero-memory generally nonlinear function, with an input-output response equal to $-d / d z_{i}\left[\ln p_{n}\left(z_{i}\right)\right]$. For Gaussian noise, this zero-memory function is linear, and the structure reduces to the expected correlation receiver. However, for non-Gaussian noise the first derivative of the logarithm of the density function is not a linear function of the input samples, and implementation of the locally optimum receiver requires knowledge of the first-order amplitude probability density function. If the outlined approach is followed, the locally optimum receiver structures for higher level hypotheses testing or for different hypotheses are easily derived. Generally, the resulting LOTR reduces to the structure that would be used for additive Gaussian noise, except that it is preceded by the zero-memory nonlinear device described above.

\section{PERFORMANCE MEASURE}

Now that the optimal receiver structure has been derived, it is of interest to determine the performance improvements that can be expected by use of this structure compared to the one in which the nonlinearity before the correlator is suboptimal or absent. Usually, the performance of a receiver is measured by the probability of error for a given signal energy. This requires that the probability density function of the test statistic be known or, alternatively, that the probability of error be bound in some manner. For the case of threshold signals, the correlator must sum many received symbols to increase the SNR for satisfactory performance. As described previously, the nonlinear device tends to suppress large amplitude variations; thus each received digit is bound. (From a practical point of view, the received symbols are bound even when the receiver is intended to be linear, owing to the finite responses of amplifiers, filters, etc.) The test statistic, therefore, is the sum of independent finite variance random variables and, based on the Central Limit Theorem, is asymptotically Gaussian distributed. Because the density function of a Gaussian random variable is completely specified by its mean and variance, the SNR at the output of the correlator may be used to express receiver performance. Hence, the improvement factor $I$ is defined as the ratio of the output SNR of the nonlinear receiver to that of the linear receiver

$$
I=\frac{\mathrm{SNR}_{N L}}{\mathrm{SNR}_{L}}=\frac{\mathrm{SNR} \text { at output of receiver with nonlinearity present }}{\text { SNR at output of receiver with nonlinearity removed }}
$$




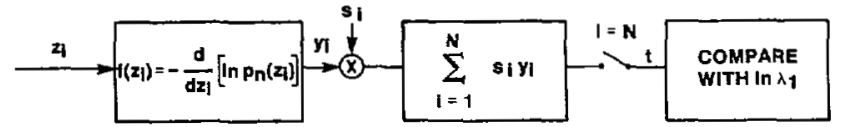

CHOOSE $H_{1}$ IF $t \geq \ln \lambda_{1}$ CHOOSE HO If $t<\ln \lambda_{1}$

Fig. 2. Block diagram of LOTR.

The general form of the improvement factor is given by [11] as

$$
I=\frac{\sigma_{n}^{2}\left(\sum_{i=1}^{N} s_{i} \bar{y}_{i}\right)^{2}}{\left(\sum_{i=1}^{N} s_{i}^{2}\right)\left(\sum_{i=1}^{N} s_{i}^{2} \operatorname{var}\left(y_{i}\right)\right)}
$$

where $\bar{y}_{i}$ and var $\left(y_{i}\right)$ are the mean and variance of the output of cases. It is canonical in nature; that is, the noise density function, which is characterized by various parameters, does not change form.

The Class B Noise Model treats noise in which the interference is highly impulsive, resulting in a noise bandwidth much greater than the front end bandwidth of the receiver. In this case, the model for the amplitude noise probability density is expressible as two distinct functions: one is valid for $|z| \leqslant$ $z_{O B}$ and the other for $|z|>z_{O B}$. The second function approaches zero much more rapidly than the first as $z$ approaches infinity. It was already pointed out that a nonlinear device operating on threshold signals in this type of noise environment will suppress large excursions in the received waveform. Thus the noise power at the output of the nonlinear device is primarily determined by the noise density function $p_{n}(z)$ for $|z|<$ $z_{c}$ where $z_{c}$ is the value at which significant suppression occurs. The more impulsive the noise environment, the more likely $z_{c}$ is smaller than $z_{O B}$. For this reason, only the first function in Middleton's model is used here. Thus

$$
p_{n}(z)=\left\{\begin{array}{cl}
\frac{1}{\pi \sqrt{\Omega}} \sum_{m=0}^{\infty} \frac{(-1)^{m} A_{\alpha}^{m}}{m !} \Gamma\left(\frac{m \alpha+1}{2}\right){ }_{1} F_{1}\left(\frac{m \alpha+1}{2} ; \frac{1}{2} ;-\frac{z^{2}}{\Omega}\right), & |z| \leqslant z_{0 B} \\
0, & |z|>z_{0 B}
\end{array}\right.
$$

of the zero-memory nonlinearity $f\left(z_{i}\right)$ and $\sigma_{n}{ }^{2}$ is the variance of the identically distributed input noise samples. The mean and variance of $y_{i}$ are defined by

$$
\begin{aligned}
\bar{y}_{i} & =\int_{-\infty}^{\infty} f\left(z_{i}\right) p_{n}\left(z_{i}-s_{i}\right) d z_{i} \\
\operatorname{var}\left(y_{i}\right) & =\overline{y_{i}^{2}}-\overline{y_{i}^{2}} \\
\overline{y_{i}{ }^{2}} & =\int_{-\infty}^{\infty} f^{2}\left(z_{i}\right) p_{n}\left(z_{i}-s_{i}\right) d z_{i} .
\end{aligned}
$$

The improvement factor for any nonlinearity and signal can be derived from (4) and (5). The improvement factors for the locally optimum threshold nonlinearity (LOTNL) $I_{O P T}$, for the clipper $I_{C}$, for the hole puncher $I_{H P}$, and for the hard limiter $I_{H L}$ are given in [11]. It should be noted that while the SNR's at the correlator output depend on the signal struc. ture, the improvement factors for the four nonlinearities are independent of the signal; this is a direct result of the smallsignal assumption.

\section{CANONICAL NOISE MODEL}

It is evident from the above discussion that the optimal receiver structure is highly dependent on the probability density function of the noise. Although various noise models have been proposed for particular environments, the only general one available to date is the Class B Noise Model suggested by Middleton [12]-[14]. This model is analytically tractable and provides excellent agreement with measured data for a variety where

$$
\begin{array}{ll}
0<\alpha<2, A_{\alpha}>0, \\
\Gamma(\cdot) & \text { gamma function, } \\
{ }_{1} F_{1}(a ; b ; x) & \text { confluent hypergeometric function, } \\
\Omega & \text { normalization factor, such that var }(z)=1 .
\end{array}
$$

Three parameters $\left(A_{\alpha}, \alpha\right.$, and $\left.z_{0 B}\right)$ define the noise model. For constant $A_{\alpha}$, the more impulsive densities (i.e., those with tails that approach zero more slowly) are characterized by smaller values of $\alpha$. In fact, the slope of the probability density function tails, plotted on a $\log -\log$ scale, is defined by the parameter $\alpha$. Although the role of $A_{\alpha}$ is not as readily apparent as that of $\alpha$, it appears to be related to the slope of the density function of the transition from the Gaussian portion to the non-Gaussian tails and to the range over which the function is Gaussian-like. The noise is Gaussian over a greater range of the density function for small values of $A_{\alpha}$. The parameter $z_{0 B}$, which is related to dynamic range in this form of the model, is the maximum value the noise can reach before saturation.

Several digital tape recordings of high-level ELF noise from the Saipan area were analyzed to yield amplitude probability distributions (APD's) [11]. These measured distributions have been compared with distributions computed from Middleton's Class B Model, and the parameters $A_{\alpha}, \alpha$, and $z_{0 B}$ have been estimated. Fig. 3 is a sample plot of the APD of typical high-level ELF noise from the Saipan area. The noise was bandpass filtered between 20 and $150 \mathrm{~Hz}$, and the distribution was measured over a 400-s time period. This APD shows the percentage of time that the magnitude of the noise exceeds the abscissa level. The APD that fits Middleton's Class 


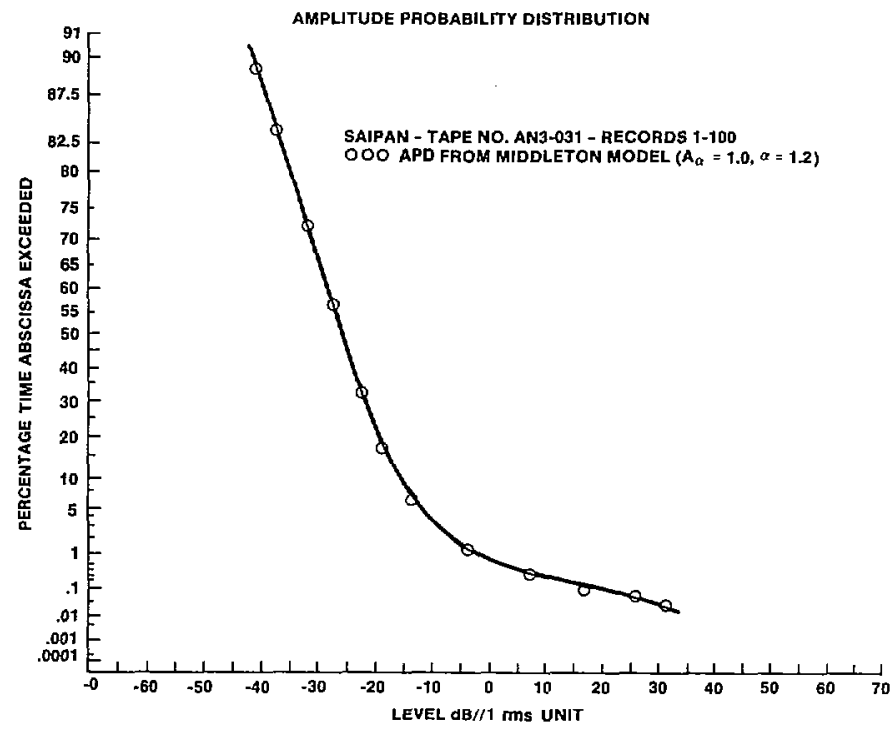

Fig. 3. APD of high-level ELF noise.

B Model was calculated, and sample points were plotted as circles on Fig. 3; the model is seen to fit the measured APD closely. From this and other comparisons, it appears that the probability density functions of high-level ELF noise can be modeled by (6), with $A_{\alpha}=1.0$ to $1.5, \alpha=1.2$ to 1.4 , and $z_{0 B}$ approaching $40 \mathrm{~dB}$.

Computation of the LOTNL for (6) is not straightforward; however, it can be approximated for small and large values of $z$ :

$$
\frac{-d}{d z}\left[\ln p_{n}(z)\right]=\left\{\begin{array}{ll}
\frac{2 z}{\Omega}, & \frac{z^{2}}{\Omega} \ll 1 \\
\frac{\alpha+1}{z}, & \frac{z^{2}}{\Omega} \gg 1
\end{array} .\right.
$$

The input-output amplitude transfer characteristic is linear for small inputs, but varies as $1 / z$ for large inputs; the larger the input pulse, the smaller the output. Fig. 4 is a plot of the complete LOTNL transfer function for the cases $A_{\alpha}=1.0$ and $\alpha=1.0$ and 0.2 . The transfer functions of the clipper and hole puncher clearly bound the LOTNL for this noise model.

\section{NONLINEAR RECEIVER PERFORMANCE}

The improvement factor of a nonlinear receiver is a function of 1) the total noise power, 2) the shape of the noise probability density function, and 3 ) the input-output characteristics of the nonlinear device. The improvement factor for the LOTNL $\left(I_{\mathrm{OPT}}\right)$ has been numerically evaluated for threshold signals and Middleton's Class B Model [11]. Figs. 5 and 6 are sample plots of the LOTNL improvement factor $\left(I_{\mathrm{OPT}}\right)$ as a function of $z_{0 B}, A_{\alpha}$, and $\alpha$. For smaller values of $A_{\alpha}$, the density function is Gaussian over a greater range of $z$, and the improvement factor depends strongly on the shape of the tails of amplitude probability density function. If the tails are decaying rapidly (larger $\alpha$ ), only modest improvements can be expected. However, if the tails approach zero very slowly (smaller $\alpha$ ), significant processing gains can be achieved. As $A_{\alpha}$ increases, the noise is Gaussian over a smaller

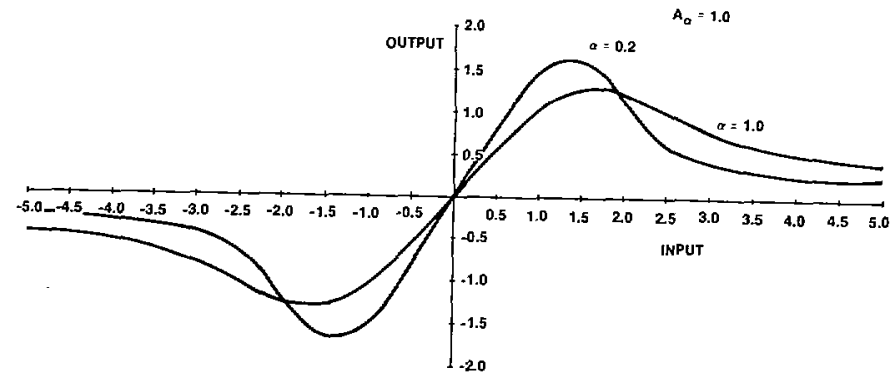

Fig. 4. Sample LOTNL input-channel characteristics for Middletown's Class B Model, where $A_{\alpha}=1.0$ and $\alpha=0.2,1.0$.

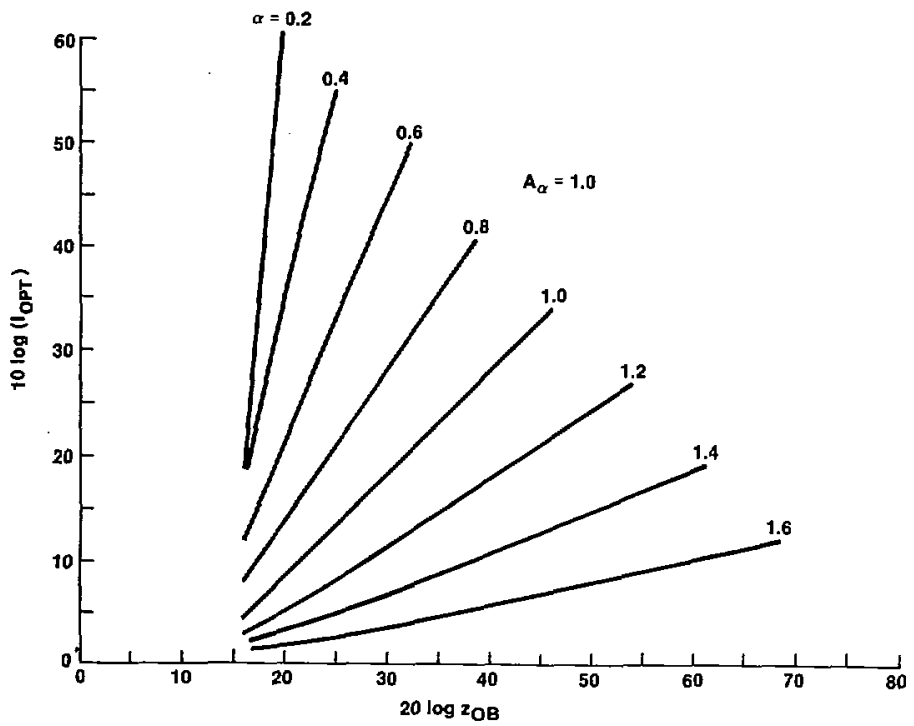

Fig. 5. Improvement factor for optimum nonlinearity, where $A_{\alpha}=1.0$ and $\alpha$ $=0.2-1.6$.

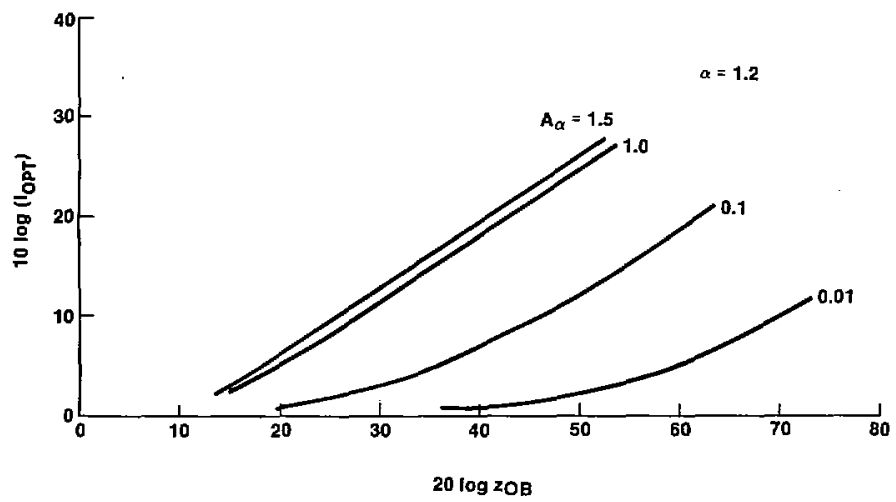

Fig. 6. Improvement factor for optimum nonlinearity, where $\alpha=1.2$ and $A_{\alpha}$ $=0.01-1.5$.

range, and improvement will occur at lower values of $z_{0 B}$. Here, the shape of the tails also greatly affects the processing gain. In general, the improvement factor increases as $z_{0 B}$ and $A_{\alpha}$ increase and as $\alpha$ decreases. For high-level ELF noise, improvements of 10-20 dB are possible with the LOTR.

Because, in practice, the LOTNL is often replaced by a clipper, hole puncher, or hard limiter, it is important to examine the performance degradations caused by the use of these simpler suboptimal devices. The improvement factors for these suboptimal nonlinearities have also been evaluated for Middleton's Class B Noise Model [11]; typical results are shown for 
the clipper $\left(I_{C}\right)$ and the hole puncher $\left(I_{H P}\right)$ in Figs. 7 through 10. The performance of the clipper and hole puncher are functions of the clip level, or the percentage of time the input is in the nonlinear portion of the device. The hard limiter is shown as a special case of the clipper in which the clip level is set to yield 100-percent clipping.

Figs. 7 and 8 show the ratio of the clipper improvement factor to optimum improvement factor versus clip percentage for various $A_{\alpha}$ and $\alpha$. While the optimum clip level (that which results in the best performance) depends on the noise parameters defining the density function, the performance of the clipper relative to the optimum nonlinearity is not a strong function of the percentage of time in which the input is in the nonlinear portion of the transfer function. In fact, if the clip level is set to obtain 90-percent clipping, clipper performance will remain within $4.5 \mathrm{~dB}$ of the optimum over a large range of noise parameters. When the absolute measures shown in Figs. 5 and 6 are used, the optimal improvement factor is large for those values of $\alpha$ where clipper performance decreases. As the clipper performance decreases due to a decreasing $\alpha$, the optimal receiver perfornance increases so that the clipper performance is always close to that of the optimal nonlinearity.

For high-level ELF noise, the clip level should be set to obtain a 40-percent clipping. This clip level yields a robust receiver performance, which is within $0.5 \mathrm{~dB}$ of the locally optimum nonlinearity and is degraded by less than a few tenths of a decibel for less impulsive ELF noise.

Even simpler than the clipper is the hard limiter, with an improvement performance $\left(I_{H L}\right)$ approaching that of the clipper without the requirement for adaptive control of the clip level. The more impulsive the noise, the closer the performance of the hard limiter approaches that of the optimum clipper. For high-level ELF noise, the use of the hard limiter will result in as much as a $1.0-\mathrm{dB}$ degradation relative to the adaptive clipper. The analytical results of this study do not confirm the larger degradations (up to $3 \mathrm{~dB}$ ) for the hard limiter compared to the optimumly adjusted clipper reported by Evans and Griffiths [4]; however, it is possible that low levels of powerline interference on some of the noise recordings used in the studies may have caused this greater degradation.

Figs. 9 and 10 show the performance degradations that result when the LOTNL is replaced by a hole puncher. An optimally adjusted hole puncher yielded a performance within $1 \mathrm{~dB}$ of the LOTNL for all of the noise parameters examined, including those that described the very impulsive noise that resulted in up to $4.5-\mathrm{dB}$ degradations for a clipper. The performance of the hole puncher is very sensitive to both the clip level and the noise parameters, and the performance degrades rapidly when the clip level is misadjusted. For high-level ELF noise, the clip level for the hole puncher should be set to obtain between a 5 and 10 hole punching percentage. With a clip level in this range, the hole puncher relative performance $I_{H P} / I_{\mathrm{OPT}}$ would vary from 0.5 to $1.5 \mathrm{~dB}$; this performance would depend on the clip level and actual parameters of the high-level ELF noise, with possible greater degradations for less impulsive ELF noise.

The receivers presently in service as part of the U.S. Navy experimental ELF program and those that will be part of the

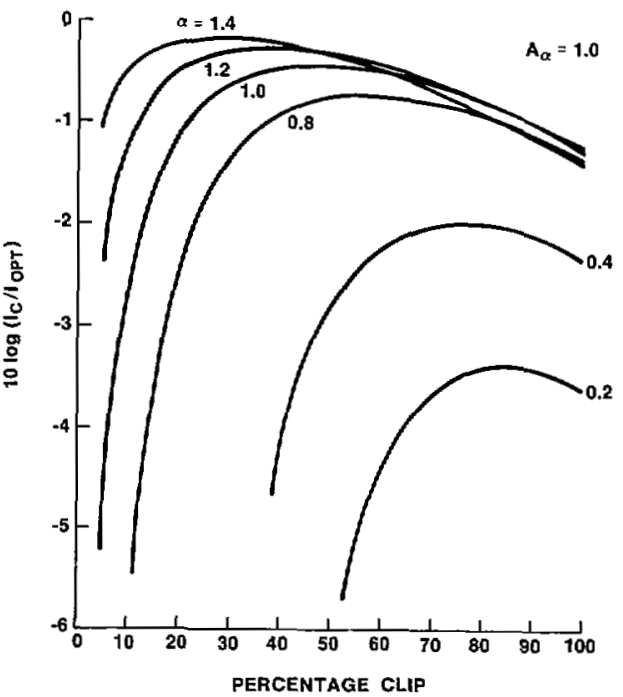

Fig. 7. Clipper performance relative to optimum performance, where $A \alpha=$ 1.0 and $\alpha=0.2-1.4$.

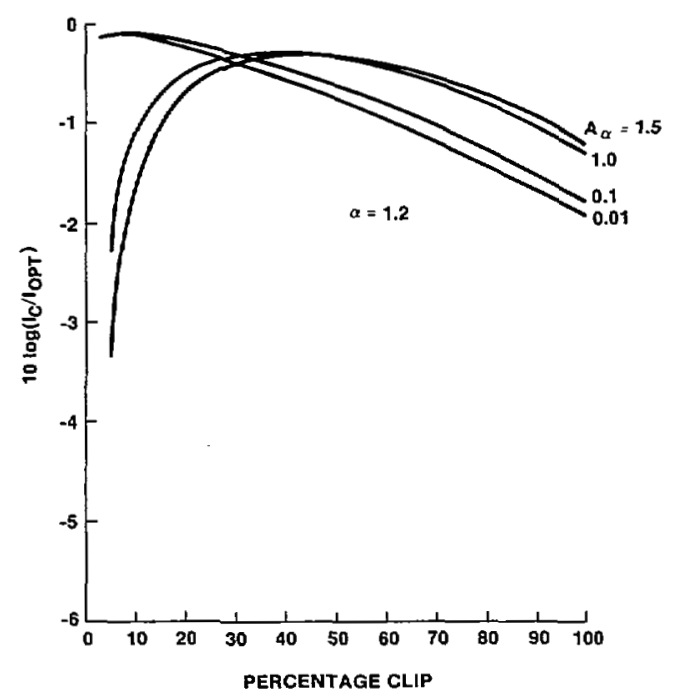

Fig. 8. Clipper performance relative to optimum performance, where $\alpha=$ 1.2 and $A_{\alpha}=0.01-1.5$.

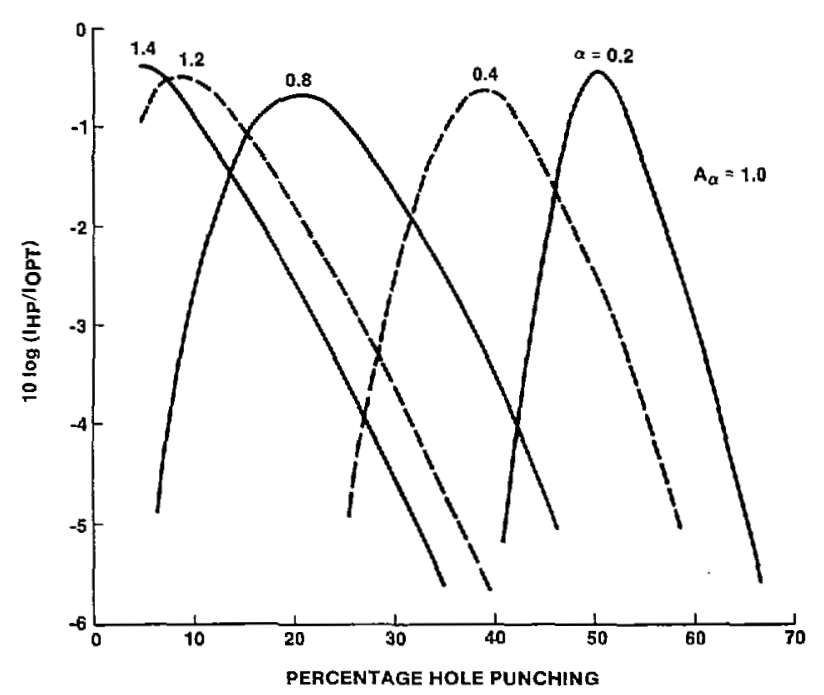

Fig. 9. Hole puncher performance relative to optimum performance, where $A_{\alpha}=1.0$ and $\alpha=0.2-1.4$ 


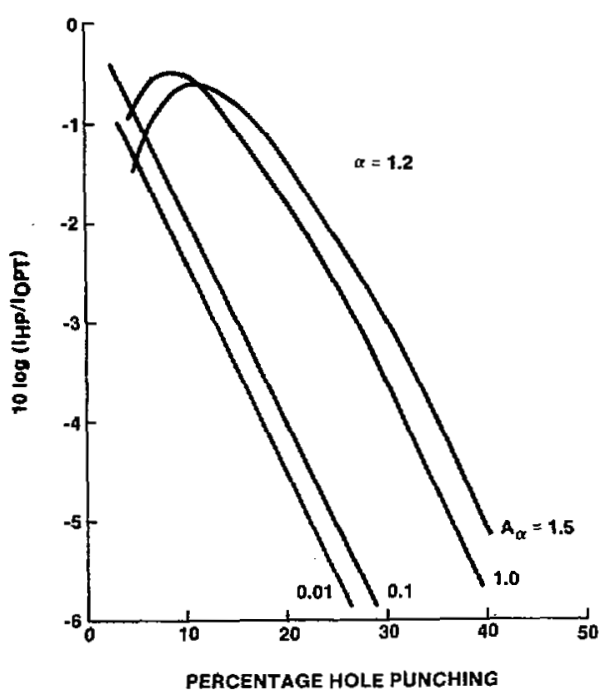

Fig. 10. Hole puncher performance relative to optimum performance, where $\alpha=1.2$ and $A_{\alpha}=0.01-1.5$.

operational ELF system under development both use an adaptive clipper as a nonlinearity before the demodulation and decoding portions of the receiver. In general, atmospheric noise samples are not independent; these samples are often corrupted by the addition of locally generated powerline interference, and individual noise spikes are "smeared" as they pass through the sea water above the receiving antenna. Such effects can reduce the improvements offered by a nonlinear receiver. To maximize the performance of the nonlinear ELF receiver, the U.S. Navy design places 1) a "whitening" filter, 2) a set of tracking notch filters to remove powerline interference, and 3) an "inverse ocean" filter before the adaptive clipper. The clip level is set, based on real time measurements, to maintain a 0.4 clip probability. Based on the results presented above, the adaptive clipper provides a more robust performance than a hole puncher and does not suffer the performance degradations of a hard limiter. The present ELF design should yield a performance within $0.5 \mathrm{~dB}$ of the performance that could be achieved with the LOTNL for high-level ELF noise. This performance is achieved without the need to estimate the noise parameters in real time.

Finally, it is important to address two of the assumptions presented in Section II. First, the choice of SNR as the parameter for comparison of the yarious nonlinearities was based on the assumption that the decision variable was the result of summing a large number $N$ of bounded non-Gaussian samples. Clearly, all the nonlinearities considered here resulted in a significant limiting of the input samples, and, in fact, the amplitude probability density function of the noise defined a bounded variable even for the "linear" receiver. For U.S. Navy ELF receivers presently in use or under development, $N$ has a value greater than $10^{5}$. Previous empirical results have shown that the decision variable is Gaussian when the sum is over only 500 clipped ELF noise samples [15].

Second, throughout this discussion signals have been assumed to be threshold without consideration of how low the input SNR had to be to produce a true threshold signal. Equation (4) has been numerically evaluated for constant level and

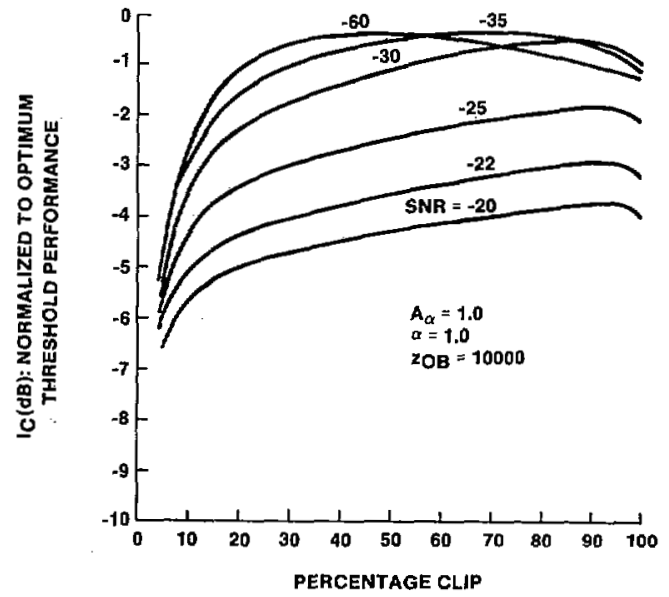

Fig. 11. Sample clipper performance as a function of input SNR.

sinusoidal signals. Fig. 11 shows a sample result of clipper performance relative to optimum performance as a function of input SNR. Because the $-60-\mathrm{dB}$ curve did not charige for smaller input SNR's, the $-60-\mathrm{dB}$ input SNR can be considered as "threshold." Higher input SNR's resulted in reduced performance. For the ELF systems of interest, the receiver input SNR will be in the range of -45 to $-55 \mathrm{~dB}$ for the high-noise (worst case) environments and, perhaps, as high as $-30 \mathrm{~dB}$ under median or normal conditions. For the highnoise case, the ELF receiver performance will be within a few tenths of a decibel of threshold performance. For higher input SNR, the improvement provided by the nonlinearity may decrease slightly if the noise parameters are assumed to remain constant; however, at these higher input SNR's, slight degradations can be easily tolerated. In fact, the higher input SNR's may correspond to less impulsive noise (i.e., smaller $A_{\alpha}$ and larger $\alpha$ ). Results similar to those in Fig. 11 for less impulsive noise indicate that nonthreshold performance degradations occur at higher input SNR's than those shown in this figure; hence, the ELF receiver performance will not suffer significantly from nonthreshold degradation.

\section{CONCLUSIONS}

The optimum receiver structure for the threshold detection of known signals in white non-Gaussian noise takes the form of a zero-memory nonlinearity, with an input-output transfer characteristic given by $-d / d x\left[\ln p_{n}(x)\right]$, followed by a correlator receiver. The performance of this receiver, as well as of those in which the nonlinearity is replaced by a clipper, hole puncher, or hard limiter has been numerically evaluated for Middleton's Class B Noise Model. The results of this work indicate that, compared to a linear receiver, the addition of the locally optimum nonlinearity will improve receiver performance by $10-20 \mathrm{~dB}$, depending on the actual characteristics of the high-level ELF noise. For the suboptimal nonlinearities considered, it is shown that the clipper is more robust than the hole puncher, because it is less sensitive to variations in the noise parameters for a given nonlinearity operating point. However, an appropriately adjusted clipper or hole puncher always provides better threshold performance than a hard limiter. The most significant conclusion is that the perform- 
ance improvements that might be obtained by the use of the locally optimum nonlinearity in comparison to an adaptive clipper, such as that presently implemented in the U.S. Navy ELF receiver, are small (on the order of $0.5 \mathrm{~dB}$ for high-level ELF noise).

\section{ACKNOWLEDGMENT}

The author wishes to thank Dr. D. Miller and Dr. W. Von Winkle for their support during these studies, and Dr. D. Middleton for the guidance provided during several discussions at NUSC. R. Houle, Ms. W. Rannenberg, R. Aiksnoras, and R. Hall also made significant contributions to various phases of the work described in this paper.

\section{REFERENCES}

[1] L. H. Ginsberg, "Extremely low frequency (ELF) atmospheric noise level statistics for project Sanguine," IEEE Trans. Commun. Technol., vol. COM-22, pp. 555-561, Apr. 1974.

[2] J. E. Evans, "Preliminary analysis of ELF noise," Lincoln Laboratory, M.I.T., Lexington, MA, Tech. Note 1969-18, DDC AD-691814, Mar. 1969.

[3] F. Horner, "Radio noise from thunderstorms," Advances in Radio Research, vol, II. New York: Academic, 1964.

[4] J. E. Evans and A. S. Griffiths, "Design of a sanguine noise processor based on worldwide extremely low frequency (ELF) recordings," IEEE Trans. Commun. Technol., vol. COM-22, pp. 528-539, Apr. 1974.

[5] A. Griffiths, "ELF noise processing," Lincoln Laboratory, M.I.T., Lexington, MA, Tech. Rep. 490, DDC AD-739907, Jan. 1972.

[6] S. L. Bernstein, D. A. McNeill, and I. Richer, "A signaling scheme and experimental receiver for extremely low frequency (ELF) communication," IEEE Trans. Commun. Technol., vol. COM-22, pp. 508-527, Apr. 1974.

[7] S. S. Rappaport and L. Kurz, "An optimal nonlinear detector for digital data transmission through non-Gaussian channels," IEEE Trans. Commun. Technol., vol. COM-14, pp. 266-274, June 1966.

[8] O. Antonov, "Optimal detection of signals in non-Gaussian noise," Radio Eng. Electron. Phys. (USSR), vol. 12, pp. 541-548, 1967.

[9] D. Middleton, "Threshold signal reception in electromagnetic interference environments, Part II: Receiver structures and performance for Class A EMI environments and scenarios," Nat. Telecommunications and Information Administration (NTIA), U.S. Dep. of Commerce, Tech. Rep. NTLA-CR-82-17, Mar. 1982.

[10] D. Middleton and A. D. Spaulding, "Optimum reception in non-
Gaussian electromagnetic interference environments, Part II: Optimum and suboptimum threshold signal detection in Class A and B noise," Nat. Telecommunications and Information Administration (NTIA), U.S. Dep. of Commerce, Tech. Rep. NTIA-83-120, Washington, DC, Jan. 1983.

[11] R. F. Ingram and R. Houle, "Performance of the optimum and several suboptimum receivers for threshold detection of known signals in additive, white non-Gaussian noise," Naval Underwater Systems Ctr., New London, CT, NUSC Tech. Rep. 6339, Nov. 1980.

[12] D. Middleton, "Statistical-physical models of man-made radio noise, Part I: First-order probability models of the instantaneous amplitude,", Office of Telecommunications, U.S. Dep. of Commerce, Tech. Rep. OT-74-36, Apr. 1974 (U.S. Gov. Printing Office, Washington, DC 20402).

[13] - Statistical physical models of man-made and natural radio noise, Part II: First-order probability models of the envelope and phase," Office of Telecommunications, U.S. Dep. of Commerce, Tech. Rep. OT76-86, Apr. 1976 (U.S. Gov. Printing Office, Washington, DC 20402).

[14] _ "Statistical-physical models of man-made and natural radio noise, Part III: First-order probability models of the instantaneous amplitude of Class B interference," Nat. Telecommunications and Information Administration (NTIA), Institute of Telecommunications Sciences, U.S. Dep. of Commerce, Tech. Rep. NTIA-CR-78-1, June 1978.

[15] J. E. Evans and A.S. Griffiths, "Design of an ELF noise processor," in Proc. Ocean 72 IEEE Conf. on Eng. in the Ocean Environment, IEEE Publ. 72 CHO 660-1 OCC, Sept. 1972.

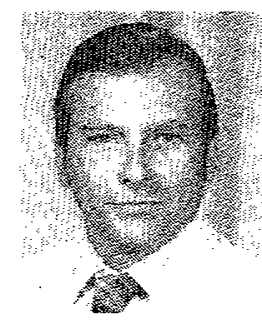

Raymond F. Ingram (S'60-M'70) was born in New London, CT, on February 26, 1941. He received the B.S. degree in electrical engineering from Northeastern University, Boston, MA, in 1964, and the M.S. degree in electrical engineering from the University of Connecticut, Storrs, in 1969 . He also studied electrical engineering at the University of Rhode Island, Kingston.

Since 1959 he has been employed at the Naval Underwater Systems Center, New London, CT, where he was appointed Systems Analysis Branch Head in the Submarine Electromagnetics System Department in 1981. His work includes signal and receiver design, systems performance predictions, jamming analyses, and analysis of direction finding techniques, particularly for low-frequency electromagnetic application.

Mr. Ingram is a member of Eta Kappa Nu. 